\title{
Increased bioavailability of hesperetin-7-glucoside compared with hesperidin results in more efficient prevention of bone loss in adult ovariectomised rats
}

\author{
Véronique Habauzit ${ }^{1}$, Inge-Lise Nielsen ${ }^{2}$, Angel Gil-Izquierdo ${ }^{3}$, Anna Trzeciakiewicz ${ }^{1}$, Christine Morand ${ }^{1}$, \\ Winnie Chee $^{2} \dagger$, Denis Barron ${ }^{2}$, Patrice Lebecque ${ }^{1}$, Marie-Jeanne Davicco ${ }^{1}$, Gary Williamson ${ }^{2}$, \\ Elizabeth Offord ${ }^{2}$, Véronique Coxam ${ }^{1}$ and Marie-Noëlle Horcajada ${ }^{1} *$ \\ ${ }^{1}$ Human Nutrition Unit, UMR 1019, ASM, INRA, Clermont-Ferrand/Theix, St-Genès Champanelle F-63122, France \\ ${ }^{2}$ Nestlé Research Center, Vers Chez Les Blanc, 1000 Lausanne 26, Switzerland \\ ${ }^{3}$ Departamento de Ciencia y Tecnología de Alimentos, CEBAS - CSIC, 30100 Espinardo, Murcia, Spain \\ (Received 3 September 2008 - Revised 9 March 2009 - Accepted 11 March 2009 - First published online 27 April 2009)
}

Hesperidin (Hp), a citrus flavonoid predominantly found in oranges, shows bone-sparing effects in ovariectomised (OVX) animals. In human subjects, the bioavailability of $\mathrm{Hp}$ can be improved by the removal of the rhamnose group to yield hesperetin-7-glucoside (H-7-glc). The aim of the present work was to test whether H-7-glc was more bioavailable and therefore more effective than Hp in the prevention of bone loss in the OVX rat. Adult 6-month-old female Wistar rats were sham operated or OVX, then pair fed for $90 \mathrm{~d}$ a casein-based diet supplemented or not with freeze-dried orange juice enriched with $\mathrm{Hp}$ or $\mathrm{H}-7$-glc at two dose equivalents of the hesperetin aglycone $(0.25$ and $0.5 \%)$. In the rats fed $0.5 \%$, a reduction in OVX-induced bone loss was observed regarding total bone mineral density (BMD): $+7.0 \%$ in OVX rats treated with Hp (HpOVX) and $+6.6 \%$ in OVX rats treated with $\mathrm{H}-7$-glc $(\mathrm{H}-7$-glcOVX) $v$. OVX controls $(P<0.05)$. In the rats fed $0.25 \%$ hesperetin equivalents, the H-7-glcOVX group showed a $6.6 \%$ improvement in total femoral BMD $v$. the OVX controls $(P<0 \cdot 05)$, whereas the Hp diet had no effect at this dose. The BMD of rats fed $0.25 \% \mathrm{H}-7-\mathrm{glc}$ was equal to that of those given $0.5 \% \mathrm{Hp}$, but was not further increased at $0.5 \% \mathrm{H}-7$-glc. Plasma hesperetin levels and relative urinary excretion were significantly enhanced in the H-7-glc $v$. Hp groups, and the metabolite profile showed the absence of eriodictyol metabolites and increased levels of hesperetin sulphates. Taken together, improved bioavailability of H-7-glc may explain the more efficient bone protection of this compound.

Bioavailability: Bone mineral density: Hesperidin: Flavanones: Rats

Nutrition plays an important role in the dietary management and prevention of osteoporosis, a major public health problem. While it is established that $\mathrm{Ca}$, vitamin $\mathrm{D}$ and micronutrients are essential for bone health, other compounds such as polyphenols, found abundantly in fruit and vegetables, show potential for bone-protective effects. For example, rodent studies have shown that quercetin and quercetin-3-rhamnoglucoside (rutin) from onions ${ }^{(1,2)}$, resveratrol from red wine ${ }^{(3)}$ and isoflavones from soya ${ }^{(4)}$ inhibit ovariectomised (OVX)induced bone loss. Until now, most of the human intervention studies have been carried out with high levels of soya isoflavones (80-90 mg), some studies showing prevention of bone loss in postmenopausal women ${ }^{(5,6)}$, while others not ${ }^{(7,8)}$. The level of consumption of soya products is rather low in Western countries compared with Asian ones, whose daily intakes average $20-40 \mathrm{mg}^{(9)}$. Therefore, it would be of interest to identify commonly consumed polyphenols with bone-protective effects, as soya is not used as a staple in the Western diet.
Hesperidin (Hp), a monomethoxylated flavanone found abundantly in citrus fruits such as oranges ${ }^{(10)}$, is highly consumed in Western countries. Indeed, in Finland, Hp consumption was estimated to be $28 \mathrm{mg} / \mathrm{d}$, contributing to $50 \%$ of total flavonoid intake ${ }^{(11)}$. Hp and metabolites show promising health benefits due to their antioxidant, anti-inflammatory and lipid-lowering properties demonstrated in preclinical models $^{(12-14)}$. Furthermore, Hp shows bone-sparing effects in OVX mice and rats of various ages, when provided in the diet at $0.5 \%(15,16)$, and citrus juice consumption has been shown to prevent bone loss in male orchidectomised rats ${ }^{(17)}$.

Flavonoids are commonly conjugated to glycosides when present in plants. Hp is one such conjugated glycoside consisting of the aglycone hesperetin ( $4^{\prime}$-methoxy- $3^{\prime}, 5,7$-trihydroxyflavanone) bound to rutinose (i.e. one molecule of rhamnose and one molecule of glucose) in the $\mathrm{C}_{7}$-position ${ }^{(18)}$. Hp has limited bioavailability in human subjects, due to the delayed action of the colonic microflora needed to release the rutinose

Abbreviations: BMD, bone mineral density; D-BMD, diaphyseal BMD; dM-BMD, distal metaphyseal BMD; DPD, deoxypyridinoline; H-7-glc, hesperetin-7glucoside; Hp, hesperidin; M-BMD, metaphyseal BMD; OC, osteocalcin; OVX, ovariectomy; pM-BMD, proximal BMD; T-BMD, total BMD.

* Corresponding author: Marie-Noëlle Horcajada, fax + 334736246 38, email horcajad@clermont.inra.fr

$\dagger$ Present address: Department of Nutrition and Dietetics, International Medical University, Plaza Komanwel, Bukit Jalil, 50700 Kuala Lumpur, Malaysia. 
moiety of $\mathrm{Hp}$ before absorption of its aglycone hesperetin. Indeed, consumption of 1 litre of orange juice containing $450 \mathrm{mg} \mathrm{Hp}$ led to circulating plasma concentrations of hesperetin of approximately $1 \mu \mathrm{M}$ with a peak at $5 \mathrm{~h}$ post-ingestion $^{(19)}$. Monoglucuronides of hesperetin were the major forms present in plasma after the ingestion of orange juice $^{(19)}$. We have recently demonstrated that enzymatic removal of the rhamnose sugar from Hp to yield hesperetin7-glucoside (H-7-glc) improves its bioavailability by 3 -fold in human subjects ${ }^{(20)}$. The removal of the terminal rhamnose sugar changes the absorption site from the colon to the small intestine, as it has been previously demonstrated with rutin and quercetin-4-glucoside in onions ${ }^{(21)}$.

The aim of the present study was to test whether H-7-glc was more bioavailable than $\mathrm{Hp}$ in the OVX rat (as in human subjects) and whether this correlated with improved efficiency in prevention of bone loss.

\section{Experimental methods}

\section{Animals and diets}

The study was carried out in accordance with the recommendations of the Regional Ethical Committee on animal experimentation in France. Fifty-eight adult, 6-month-old virgin female Wistar rats were purchased from Institut National de la Recherche Agronomique (Clermont-Ferrand/Theix, St-Genès Champanelle, France). Five groups of ten rats were surgically OVX and one group of eight rats was sham operated (SH) under anaesthesia, using chloral hydrate (Fluka Chemie AG, Buchs, Switzerland; $80 \mathrm{~g} / \mathrm{l}$ in saline solution; $0.4 \mathrm{ml} / 100 \mathrm{~g}$ body weight intraperitonealy). The animals were housed individually in plastic cages allowing separation and collection of urine and faeces at $21^{\circ} \mathrm{C}$ with relative humidity of $55 \%$ and under a $12 \mathrm{~h}: 12 \mathrm{~h}$ light:dark cycle.

The animals were fed a semipurified standard diet devoid of any soya proteins (Institut National de la Recherche Agronomique, Jouy en Josas, France). The control diet contained $150 \mathrm{~g}$ casein $/ \mathrm{kg}, \quad 50 \mathrm{~g}$ rapeseed oil $/ \mathrm{kg}, \quad 16.8 \mathrm{~g}$ $\mathrm{Ca}_{3} \mathrm{PO}_{4}\left(2 \mathrm{H}_{2} \mathrm{O}\right) / \mathrm{kg}, 6.5 \mathrm{~g} \mathrm{NaCl} / \mathrm{kg}, 11.4 \mathrm{~g}$ potassium citrate/ $\mathrm{kg}, 5 \mathrm{~g} \mathrm{MgSO}_{4}\left(7 \mathrm{H}_{2} \mathrm{O}\right) / \mathrm{kg}, 10 \mathrm{~g}$ trace elements mix $/ \mathrm{kg}, 10 \mathrm{~g}$ vitamin $\mathrm{mix} / \mathrm{kg}$ and wheat starch quantum satis to achieve $1 \mathrm{~kg}$ (here $740 \mathrm{~g} / \mathrm{kg}$ ). The $\mathrm{SH}$ group and one OVX group were fed this non-supplemented control diet. The four remaining OVX groups were given the supplemented diets prepared on the same basis as the control diet by adding $2.5 \mathrm{~g} / \mathrm{kg}$ or $5 \mathrm{~g} /$ $\mathrm{kg}$ of $\mathrm{Hp}$ or $\mathrm{H}-7-\mathrm{glc}$, respectively, at the expense of wheat starch. Hp and H-7-glc were prepared by fortification of Nestlé Thailand orange juice with $9 \cdot 2 \mathrm{~g} / \mathrm{l}$ of orange bioflavonoid complex (Nutrafur, Alcantarilla, Spain) containing $90 \% \mathrm{Hp}$, incubation with or without $\alpha$-rhamnosidase (Hesperidinase Amans concentrated, Amano Enzymes, Chipping Norton, Oxfordshire, UK) and freeze drying according to Nielsen et al. ${ }^{(20)}$. The freeze-dried juice-based product obtained without $\alpha$-rhamnosidase contained $33 \mathrm{mg} / \mathrm{g}$ of $\mathrm{Hp}$ and the product obtained with $\alpha$-rhamnosidase contained corresponding amounts of $\mathrm{H}-7$-glc. Four diets containing $0.25 \%$ w/w or $0.5 \%$ w/w of $\mathrm{Hp}$ or $\mathrm{H}-7$-glc, respectively, were prepared every week and stored at $4^{\circ} \mathrm{C}$ until use.

During the whole experimental period and in order to prevent well-known castration-induced hyperphagia, the quantity of diet given to each rat per day was adjusted to the mean food intake registered in $\mathrm{SH}$ animals (pair feeding). The animals had free access to water during the entire study, and their body weights and food intake were measured weekly. Urine of each animal was collected over a 24-h period on day 0 and the day before killing, to measure urinary excretion of deoxypyridinoline (DPD), a marker for bone resorption.

At killing, on day 90, blood samples were collected into icecooled heparinised plastic tubes containing 200 peptidase inhibitory units of aprotinin (Iniprol, Choay, Paris, France) per millilitre blood, and centrifuged immediately $(3500 \mathrm{~g}$ for $5 \mathrm{~min}$ at $4^{\circ} \mathrm{C}$ ). Then plasma samples were frozen at $-20^{\circ} \mathrm{C}$ until measurements of osteocalcin (OC), a marker of osteoblastic activity. Before freezing at $-20^{\circ} \mathrm{C}$, plasma samples dedicated to the analysis of hesperetin metabolites were acidified with acetic acid $(0.1 \mathrm{M}, 1 \%)$ in order to protect the structure of these metabolites. Uterine horns were removed and immediately weighed. Femurs were separated from adjacent tissue, cleaned and used for physical measurements (mechanical testing and bone mineral density (BMD)).

\section{Bone measurements}

Bone mineral density. BMD was assessed by dual-energy X-ray absorptiometry, with the Hologic QDR-4500 A X-ray bone densitometer. The total right femur BMD (T-BMD), as well as the BMD of two subregions, one corresponding to the metaphyseal zone (M-BMD), rich in cancellous bone, and the other to the diaphyseal zone (D-BMD), rich in cortical bone, were determined ${ }^{(22)}$. In each femur scan, distal (dM-BMD) and proximal (pM-BMD) metaphyseal regions of interest were positioned at the same distance from the distal and proximal femur extremity, respectively, and with the same height. The region of interest between the two preliminary delimited metaphyseal zones corresponds to the diaphyseal subregion. Results are given in $\mathrm{g} / \mathrm{cm}^{2}$.

Femoral mechanical testing. Immediately after collection in $\mathrm{NaCl}(9 \mathrm{~g} / \mathrm{l})$, the length of the left femur and the mean diameter of the diaphysis (i.e. the mean of the greatest and the smallest diaphysis diameters) were measured using a precision caliper (Mitutoyo, Shropshire, UK). Femoral failure load was determined using a three-point bending test with a Universal Testing Machine (Instron 4501; Instron, Canton, MA, USA). The load at rupture (N) was automatically determined and recorded by Instron 4501 software. This method was previously validated by using Plexiglas standard probes $^{(23)}$.

Bone biomarkers analyses. Plasma OC concentrations ( $\mathrm{ng} / \mathrm{ml})$ were measured by RIA, using rat ${ }^{125} \mathrm{I}$-labelled OC, goat anti-rat OC antibody and donkey anti-goat second antibody (Biochemical Technologies kit, Stoughton, MA, USA). The sensitivity was $0.01 \mathrm{ng} / \mathrm{ml}$. The intra- and interassay precisions were 6.8 and $8.9 \%$, respectively.

DPD was measured in urine by competitive RIA (Pyrilinks D kit; Metra Biosystems, Mountain View, CA, USA). The assay requires a rat monoclonal antibody against DPD, which is coated to the inner surface of a polystyrene tube and ${ }^{125} \mathrm{I}$-labelled DPD. In the present experimental conditions, the sensitivity was $2 \mathrm{nmol} / \mathrm{l}$, and the intra- and interassay variations were 4.0 and $6.0 \%$, respectively. Results are expressed as nmol of $\mathrm{DPD} / \mathrm{mmol}$ of creatinine to take into account 
interindividual differences of urine concentration ${ }^{(24)}$. The creatinine assay kit (BioMérieux, Marcy l'étoile, France) is based on a modified Jaffe's method in which picric acid forms a yellow compound in presence of creatinine ${ }^{(25)}$.

\section{Leptin assay}

Plasma leptin concentration was assessed by RIA using antirat leptin antibody and rat leptin as a standard (Rat Leptin RIA kit; Linco Research, Inc., St Charles, MO, USA). The lowest limit of sensitivity was $0.5 \mathrm{ng} / \mathrm{ml}$, and the intra- and interassay variations were 1.5 and $2.5 \%$, respectively.

\section{Analysis of hesperetin and metabolites in plasma and urine}

Preparation of plasma and urine samples for HPLC analyses. HPLC analyses were performed directly or after an hydrolysis step for which plasma samples $(180 \mu \mathrm{l})$ were acidified to $\mathrm{pH} 4.9$ with acetic acid, incubated for $5 \mathrm{~h}$ at $37^{\circ} \mathrm{C}$ with $\beta$-glucuronidase (1000 units) and sulphatase (forty-five units; from Helix pomatia, Sigma G0876, L'Isle d'Abeau, Chesnes, France), mixed with four volumes of methanol-HCl $(0 \cdot 2 \mathrm{M})$ and the supernatant analysed after centrifugation (4 min, $14000 \mathrm{rpm})$. Urine samples were diluted in acetate buffer $(0 \cdot 1 \mathrm{M}, \mathrm{pH} 4 \cdot 9)$, mixed with four volumes of methanol- $\mathrm{HCl}$ $(0.2 \mathrm{M})$ and the supernatant analysed after centrifugation as mentioned earlier.

Analysis of hesperetin metabolites in plasma by HPLC. Qualitative analysis of hesperetin metabolites and determination of the respective proportion of hesperetin conjugates (percentage of total flavanones) were performed in non-hydrolysed plasma by HPLC with mass spectrometric detection. Plasma and urine hesperetin concentrations were obtained after the hydrolysis step described earlier and analysis by HPLC with detection by CoulArray. For HPLC with mass spectrometric detection, a YMC-Pack ODS-AM column $(250 \times 3.0 \mathrm{~mm}$ inner diameter, $5 \mu \mathrm{m}$ particle size $)$ was eluted with water-formic acid $(99: 1, \mathrm{v} / \mathrm{v}$; solvent $\mathrm{A})$ and acetonitrile-formic acid $(99: 1, \mathrm{v} / \mathrm{v}$; solvent B): $20 \% \mathrm{~B}$ for $2 \mathrm{~min}$; linear gradient to $45 \%$ of $\mathrm{B}$ over $20 \mathrm{~min}$; $65 \%$ of $\mathrm{B}$ from 20 to $30 \mathrm{~min}$. Detection was by a diode array detector and API 2000 mass spectrometer. Liquid chromatographyelectrospray ionisation-MS/MS triple quadrupole MS was optimised for hesperetin, hesperetin 7-O-glucuronide and homoeriodictyol. $\mathrm{MS}$ and $\mathrm{MS}^{2}$ data were acquired in the multiple-reaction monitoring mode, with maximum intensity for the transitions at $\mathrm{m} / \mathrm{z}$ 301:286 (negative mode), 477:301 (negative mode) and 303:151 (positive mode), respectively. Hesperetin 7-O-glucuronide was confirmed using an authentic synthetic standard.

For multichannel electrochemical detection, HPLC analysis was performed on a $150 \times 4.6 \mathrm{~mm}$ Hypersil $\mathrm{BDS} \mathrm{C}_{18}-5 \mu \mathrm{m}$ column (Touzard et Matignon, Les Ulis, France), with an eight-channel CoulArray detector (model 5600, Eurosep, Cergy, France). Mobile phases were $30 \mathrm{~mm} \mathrm{NaH}{ }_{3} \mathrm{PO}_{4}$ buffer (pH 3) containing $20 \%$ acetonitrile (A) or $40 \%$ acetonitrile (B). Separation was achieved using a gradient elution $\left(35^{\circ} \mathrm{C}\right.$, $0.8 \mathrm{ml} / \mathrm{min}$ ): $0-3 \mathrm{~min}, 100 \% \mathrm{~A} ; 3-30 \mathrm{~min}$, linear gradient from $100 \% \mathrm{~A}$ to $100 \% \mathrm{~B} ; 30-35 \mathrm{~min}, 100 \% \mathrm{~B} ; 35.01-$ $45 \mathrm{~min}, 100 \% \mathrm{~A}$. Potentials were set at 50-350-480-550$700-760-820-850 \mathrm{mV}$. Hesperetin was quantified using the sums of height obtained on the electrodes 2,3 and 4, 5 and 6 , respectively.

\section{Statistical analysis}

Results are expressed as means with their standard errors. All data were analysed using the Graphpad Instat software (Microsoft, San Diego, CA, USA). A parametric one-way ANOVA was first performed to test for any significant difference among groups. If the results were significant $(P<0 \cdot 05)$, the Student-Newman-Keuls multiple comparisons test was used to determine the specific differences between means. Parametric ANOVA was performed when data were sampled from populations with equal variance. If not, a Kruskall-Wallis test followed by the Mann-Whitney $U$ test was performed to compare specific differences between groups. The level of significance was set at $P<0.05$ for all statistical tests.

\section{Results \\ Body and uterine weight changes}

Successful ovariectomy was confirmed by marked atrophy of uterine horns since the mean uterine weight was lower in the OVX groups compared to sham-operated $(\mathrm{SH})$ groups $(P<0.001 ;$ Table 1). No uterotrophic effect was elicited by consumption of $\mathrm{Hp}$ or $\mathrm{H}-7$-glc, since uterine weight was

Table 1. Effects of ovariectomy (OVX), hesperidin $(\mathrm{Hp})$ and hesperetin-7-glucoside ( $\mathrm{H}-7-\mathrm{glc})$ on food consumption (g/d), body and uterus weights ( $\mathrm{g}$ ) and plasma leptin $(\mathrm{ng} / \mathrm{ml})$

(Mean values with their standard errors for eight rats in sham operated $(\mathrm{SH})$ control group and ten rats per group in all other groups)

\begin{tabular}{|c|c|c|c|c|c|c|c|c|c|c|c|c|}
\hline & \multicolumn{2}{|c|}{$\mathrm{SH}$} & \multicolumn{2}{|c|}{ OVX } & \multicolumn{2}{|c|}{$0.25 \mathrm{HpOVX}$} & \multicolumn{2}{|c|}{$0.5 \mathrm{HpOVX}$} & \multicolumn{2}{|c|}{0.25 H-7-glcOVX } & \multicolumn{2}{|c|}{$0.5 \mathrm{H}-7-\mathrm{glcOVX}$} \\
\hline & Mean & SEM & Mean & SEM & Mean & SEM & Mean & SEM & Mean & SEM & Mean & SEM \\
\hline Food intake, day 30 & $16 \cdot 33$ & 0.51 & 16.55 & 0.54 & $17 \cdot 16$ & 0.32 & $17 \cdot 55$ & 0.02 & $17 \cdot 44$ & 0.23 & $17 \cdot 34$ & 0.35 \\
\hline Food intake, day 60 & $15 \cdot 74$ & 0.62 & $16 \cdot 83$ & 0.46 & 16.93 & 0.39 & 17.52 & $0 \cdot 11$ & $15 \cdot 79$ & 1.53 & $17 \cdot 06$ & 0.48 \\
\hline Initial body weight & $277 \cdot 4$ & $5 \cdot 6$ & $274 \cdot 2$ & $6 \cdot 1$ & $271 \cdot 3$ & $5 \cdot 3$ & $284 \cdot 2$ & $6 \cdot 1$ & $278 \cdot 4$ & $4 \cdot 8$ & $282 \cdot 1$ & $6 \cdot 5$ \\
\hline Final body weight & $309 \cdot 6^{\star}$ & $11 \cdot 0$ & $356 \cdot 2$ & $7 \cdot 6$ & 351.8 & $5 \cdot 7$ & 358.2 & $9 \cdot 7$ & $366 \cdot 2$ & $6 \cdot 8$ & $371 \cdot 1$ & $9 \cdot 9$ \\
\hline Uterus weight & 0.689 & 0.056 & $0.116 \dagger$ & 0.011 & $0.102 \dagger$ & 0.006 & $0.133 \dagger$ & 0.030 & $0.131 \dagger$ & 0.017 & $0.115 \dagger$ & 0.013 \\
\hline Plasma leptin & 8.07 & 1.5 & 11.41 & 0.96 & $10 \cdot 64$ & 0.72 & $9 \cdot 50$ & $1 \cdot 12$ & 11.07 & 1.08 & $12 \cdot 76$ & 0.89 \\
\hline
\end{tabular}

* Mean values were significantly different from those of all OVX groups $(P<0.01)$.

$\dagger$ Mean values were significantly different from those of $\mathrm{SH}$ control group $(P<0.001)$. 
unaffected by these treatments at both doses and was similar to that of the OVX control rats (Table 1).

Despite the pair feeding (during the whole experimental period, the daily mean food intake in each group was 16.9 (SEM 0.17) g), a significantly lower weight in SH animals was reported $(P<0 \cdot 01)$, from day 21 up to the end of the experimental period (day 90). No differences in body weight among the control and treated OVX groups were observed (Table 1). Furthermore, no significant difference in plasma leptin levels between groups was detected at day 90 (Table 1). Thus, either $\mathrm{Hp}$ or $\mathrm{H}-7-\mathrm{glc}$ consumption, at either dose, influenced neither the body weight nor the body composition, as suggested by leptin assay.

\section{Bone mineral density}

Concerning BMD, similar patterns were observed for T-BMD, D-BMD, pM-BMD and distal metaphyseal (dM-BMD) femoral mineral densities. As expected, ovariectomy induced a significant decrease in BMD at all femoral sites in OVX control rats (T-BMD: OVX control: $-7.4 \%$ v. $\mathrm{SH}, P<0 \cdot 01$, Fig. 1; data not shown for D-BMD, pM-BMD and dM-BMD).

Consumption of $0.5 \% \mathrm{Hp}$ led to a significant improvement in T-BMD in the HpOVX group compared with OVX controls (T-BMD: $+7.0 \%$ v. OVX, $P<0.05$; Fig. 1 ). In other bone regions, similar levels of improvement in BMD were observed with this dose of $\mathrm{Hp}$ (D-BMD: +6.7\%; dM-BMD: +7.0\%; and pM-BMD: $+7.9 \%$ v. OVX, $P<0.05$; data not shown). The lower Hp dose $(0 \cdot 25 \%)$ failed to prevent the significant ovariectomy-induced loss of BMD (compared with $\mathrm{SH}$ control group), as no differences were observed at any of the bone sites compared to OVX control group.

In the H-7-glcOVX groups, T-BMD was significantly improved (compared with OVX control group) at both doses of $0.25 \%$ (T-BMD: $+6.6 \%, P<0.05$; Fig. 1) and $0.5 \%$ (T-BMD: $+6.6 \%, P<0.05$; Fig. 1). BMD at diaphyseal and metaphyseal femoral sites was increased to the same extent than T-BMD with dose of $0.25 \%$ (D-BMD: $+7.0 \%, P<0.01$; dM-BMD: $+6.9 \%$; pM-BMD: $+6.9 \%, P<0.05$; data not shown) as well as dose of $0.5 \%$ (D-BMD: $+7.8 \%, P<0.01$; dM-BMD: $+6.1 \%$; pM-BMD: $+7.0 \%, P<0.05$; data not shown). Actually, compared with OVX controls, the $0.25 \%$

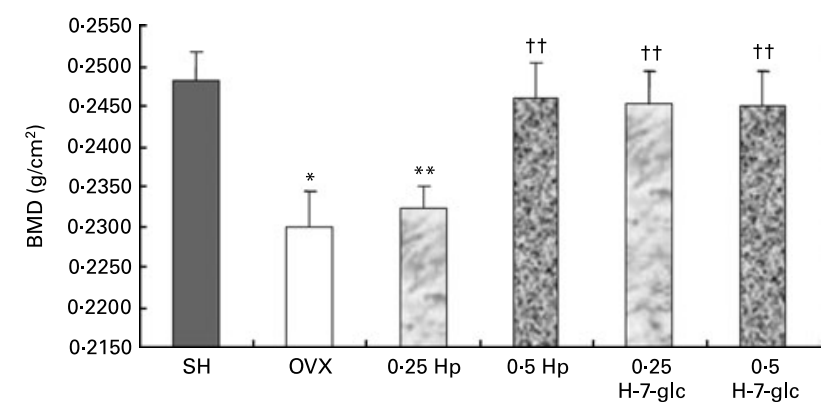

Fig. 1. Effects of ovariectomy (OVX), hesperidin (Hp) and hesperetin-7-glucoside ( $\mathrm{H}-7$-glc) on total femoral BMD $\left(\mathrm{g} / \mathrm{cm}^{2}\right)$, measured on day 90 . Results are expressed as means with their standard errors per group. * Mean values were significantly different from those of $\mathrm{SH}$ control group $(P<0.01)$. ${ }^{* *}$ Mean values were significantly different from those of $\mathrm{SH}$ control group $(P<0.05)$. ††Mean values were significantly different from those of OVX control group $(P<0.05)$. dose H-7-glcOVX group showed an equivalent improvement in BMD to that of the $0.5 \%$ dose HpOVX group and achieved the same T-BMD (Fig. 1), D-BMD or M-BMD (data not shown). However, no further increase in T-BMD, DBMD or M-BMD was seen with the higher dose of H-7-glc $(0.5 \%)$ compared to the lower one $(0 \cdot 25 \%)$. Thus, H7-glc exhibited similar BMD-sparing effects to $\mathrm{Hp}$ but at a lower dose.

\section{Bone size and ultimate load}

No significant differences among groups were demonstrated for femoral length and femoral diameter at day 90 (Table 2). Femoral strength was assessed by the femoral failure load. Ovariectomy significantly decreased this parameter compared with SH animals (OVX: $-6.5 \%$ v. SH, $P<0.05$; Table 2). Consumption of $\mathrm{Hp}$ at $0.5 \%$ inhibited this OVX-induced loss of strength (0.5\% HpOVX: $+10.0 \%$ v. OVX, $P<0.05$; Table 2). The magnitude of the effect (percentage of change) was quite similar for the two groups fed with H-7-glc $(0.25 \%$ H-7-glcOVX: $+7.4 \%$ v. OVX, $P<0.05$; $0.5 \%$ H-7-glcOVX: $+14.7 \%$ v. OVX, $P<0.05$; Table 2). In the $0.25 \% \mathrm{Hp}$ group, there was a trend to increase femoral strength, however, without reaching significance (Table 2). Thus, as shown for BMD mentioned earlier, both $\mathrm{Hp}$ and H-7-glc helped to restore the OVX-induced loss of femoral strength, but H-7-glc achieved the same effect as Hp (0.5\%) at the lower dose $(0 \cdot 25 \%)$.

\section{Bone turnover markers}

Ovariectomy resulted in a significant increase in OC plasma levels in control rats (OVX control: $+62.5 \%$ v. $\mathrm{SH}$, $P<0.001)$, probably due to enhanced bone turnover following ovariectomy, and this level was unaffected by the consumption of Hp or H-7-glc at either dose (Fig. 2(a)).

An increase in urinary DPD excretion was observed following ovariectomy (OVX control: $+145 \%$ v. $\mathrm{SH}$, $P<0 \cdot 001)$, which was partially, but significantly, inhibited by $\mathrm{Hp}$ and $\mathrm{H}-7$-glc consumption $(0.25 \% \mathrm{HpOVX}$ : $-33.0 \%$ v. OVX, $P<0.05 ; 0.5 \%$ HpOVX: $-41.7 \%$ v. OVX, $P<0.01 ; 0.25 \%$ H-7-glcOVX: $-48.4 \%$ v. OVX, $P<0.01$; $0.5 \%$ H-7-glcOVX: $-29.4 \%$ v. OVX, $P<0.05$; Fig. 2(b)).

\section{Hesperetin analyses and metabolite profile in plasma and urine}

Plasma and urine concentrations (compared with food intake). Total hesperetin concentrations in plasma, measured by multichannel electrochemical detection after an enzymatic hydrolysis, are shown in Fig. 3(a). A dose-dependent increase was observed between 0.25 and $0.5 \%$ doses of the individual treatments (i.e. $1.13 \mu \mathrm{M}$ from $0.25 \% \mathrm{Hp} ; 2.06 \mu \mathrm{M}$ from $0.5 \% \mathrm{Hp}$; $2.30 \mu \mathrm{M}$ from $0.25 \% \mathrm{H}-7$-glc; and $4.91 \mu \mathrm{M}$ from $0.5 \% \mathrm{H} 7 \mathrm{glc}$ ). However, intake of $\mathrm{H}-7$-glc at either dose led to higher (approximately 2-fold) circulating plasma levels of hesperetin than with equivalent doses of Hp. Indeed, similar plasma hesperetin concentrations were observed with $0.25 \%$ H-7-glc $(2.30 \mu \mathrm{M})$ as $0.5 \% \mathrm{Hp}(2.06 \mu \mathrm{M}$; Fig. 3(a)).

Relative urinary excretion (Fig. 3(b)) increased between the 0.25 and $0.5 \% \mathrm{Hp}$ doses (relative increase from 2.4 to $3.6 \%$ ), 
Table 2. Effects of ovariectomy (OVX), hesperidin ( $\mathrm{Hp})$, hesperetin-7-glucoside (H-7-glc) on femoral length ( $\mathrm{mm})$, femoral mean diameter ( $\mathrm{mm})$ and femoral failure load $(\mathrm{N})$ measured on day 90

(Mean values with their standard errors for eight rats in sham operated $(\mathrm{SH})$ control group and ten rats per group in all other groups)

\begin{tabular}{|c|c|c|c|c|c|c|c|c|c|c|c|c|}
\hline & \multicolumn{2}{|c|}{$\mathrm{SH}$} & \multicolumn{2}{|c|}{ ovX } & \multicolumn{2}{|c|}{$0.25 \mathrm{HpOVX}$} & \multicolumn{2}{|c|}{$0.5 \mathrm{HpOVX}$} & \multicolumn{2}{|c|}{$\begin{array}{l}0.25 \mathrm{H}-7- \\
\text { glcOVX }\end{array}$} & \multicolumn{2}{|c|}{$0.5 \mathrm{H}-7$-glcOVX } \\
\hline & Mean & SEM & Mean & SEM & Mean & SEM & Mean & SEM & Mean & SEM & Mean & SEM \\
\hline Length & 35.95 & 0.30 & $35 \cdot 70$ & 0.22 & $36 \cdot 33$ & 0.24 & 36.92 & 0.27 & $36 \cdot 34$ & 0.33 & $36 \cdot 89$ & 0.35 \\
\hline Diameter & 3.63 & 0.04 & $3 \cdot 61$ & 0.05 & 3.56 & 0.03 & 3.72 & 0.06 & 3.72 & 0.05 & 3.74 & 0.05 \\
\hline Femoral failure load & 111.3 & $3 \cdot 2$ & $104 \cdot 1^{*}$ & $2 \cdot 7$ & $108 \cdot 7$ & $3 \cdot 1$ & $114.5 \dagger$ & 3.9 & $111.8 \dagger$ & $2 \cdot 4$ & $119.4 \dagger$ & $5 \cdot 1$ \\
\hline
\end{tabular}

${ }^{*}$ Mean values were significantly different from those of $\mathrm{SH}$ control group $(P<0.05)$.

† Mean values were significantly different from those of OVX control group $(P<0.05)$

but was significantly higher in the $0.25 \% \mathrm{H}-7$-glc group $(10.9$ in $0.25 \% \mathrm{H}-7$-glc v. 2.4 in $0.25 \% \mathrm{Hp}$ ) and did not further change in the $0.5 \% \mathrm{H}$-7-glc group (10.8\%). Comparing equivalent doses of $\mathrm{Hp}$ and $\mathrm{H}-7$-glc, it was clear that the $\alpha$-rhamnosidase treatment of orange juice converting $\mathrm{Hp}$ to $\mathrm{H}$-7-glc clearly resulted in enhanced relative urinary excretion of hesperetin, indicative of increased absorption.

Metabolic profile. In all plasma samples, hesperetin-7- $O$ glucuronide and two hesperetin sulphate conjugates were detected (Fig. 4). Quantitatively (results are expressed in
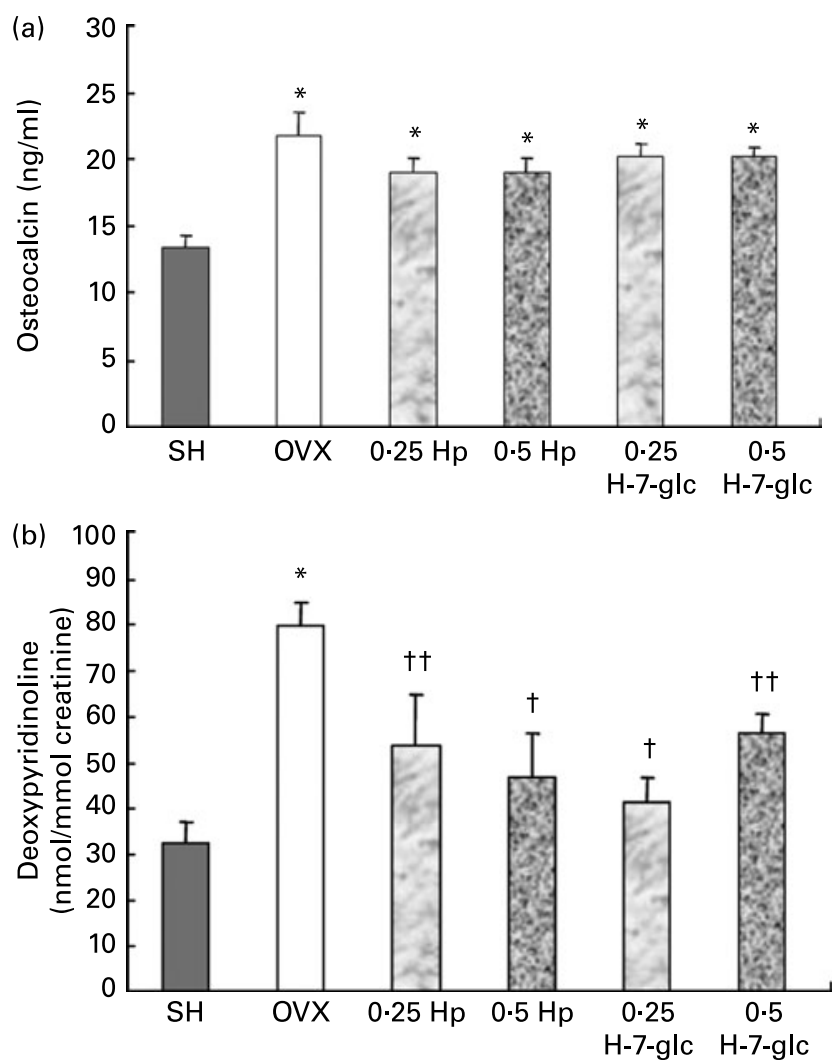

Fig. 2. Effects of ovariectomy (OVX), hesperidin $(\mathrm{Hp})$ and hesperetin-7-glucoside (H-7-glc) on (a) plasma osteocalcin ( $\mathrm{ng} / \mathrm{ml})$ and (b) urinary deoxypyridinoline $(\mathrm{nmol} / \mathrm{mmol})$, measured on day 90 . Results are expressed as means with their standard errors per group. ${ }^{*}$ Mean values were significantly different from those of $\mathrm{SH}$ control group $(P<0.001)$ † Mean values were significantly different from those of OVX control group $(P<0.01)$. ††Mean values were significantly different from those of OVX control group $(P<0.05)$. percentage of total flavanone metabolites), hesperetin 7-O-glucuronide was the major circulating form in plasma whatever the treatment or dose (54-66\%; Table 3). Conjugates with glycine and glutathione were not present, and free hesperetin aglycone was present only at very low levels in all samples.

For rats fed $\mathrm{Hp}$ in untreated orange juice, hesperetin- $3^{\prime}-O$ glucuronide, homoeriodictyol-4'-O-glucuronide and homoeriodictyol-7-O-glucuronide (all tentatively identified using MS fragmentation pattern of the aglycone standards) were observed in plasma. These compounds have been detected previously at similar levels ${ }^{(26)}$. Total homoeriodictyol glucuronides $(38.0$ and $36.9 \%$ for both 0.25 and $0.5 \%$ doses, respectively; Table 3 ) were the second most abundant metabolite group in rats fed Hp. For rats fed H-7-glc in orange juice


Fig. 3. (a) Plasma hesperetin concentrations ( $\mu \mathrm{M})$ and (b) relative urinary excretion of hesperetin (\%) measured on day 90 in ovariectomised (OVX) groups supplemented with hesperidin $(\mathrm{Hp})$ or hesperetin-7-glucoside $(\mathrm{H}-7$-glc) at both doses 0.25 and $0.5 \%$. Results are expressed as means with their standard errors per group. * Mean values were significantly different from those of all other groups $(P<0.01)$. ††Mean values were significantly different from those of the $0.25 \mathrm{HpOVX}$ group $(P<0.05)$. 㧊 Mean values were significantly different from those of HpOVX groups $(P<0.001)$. 


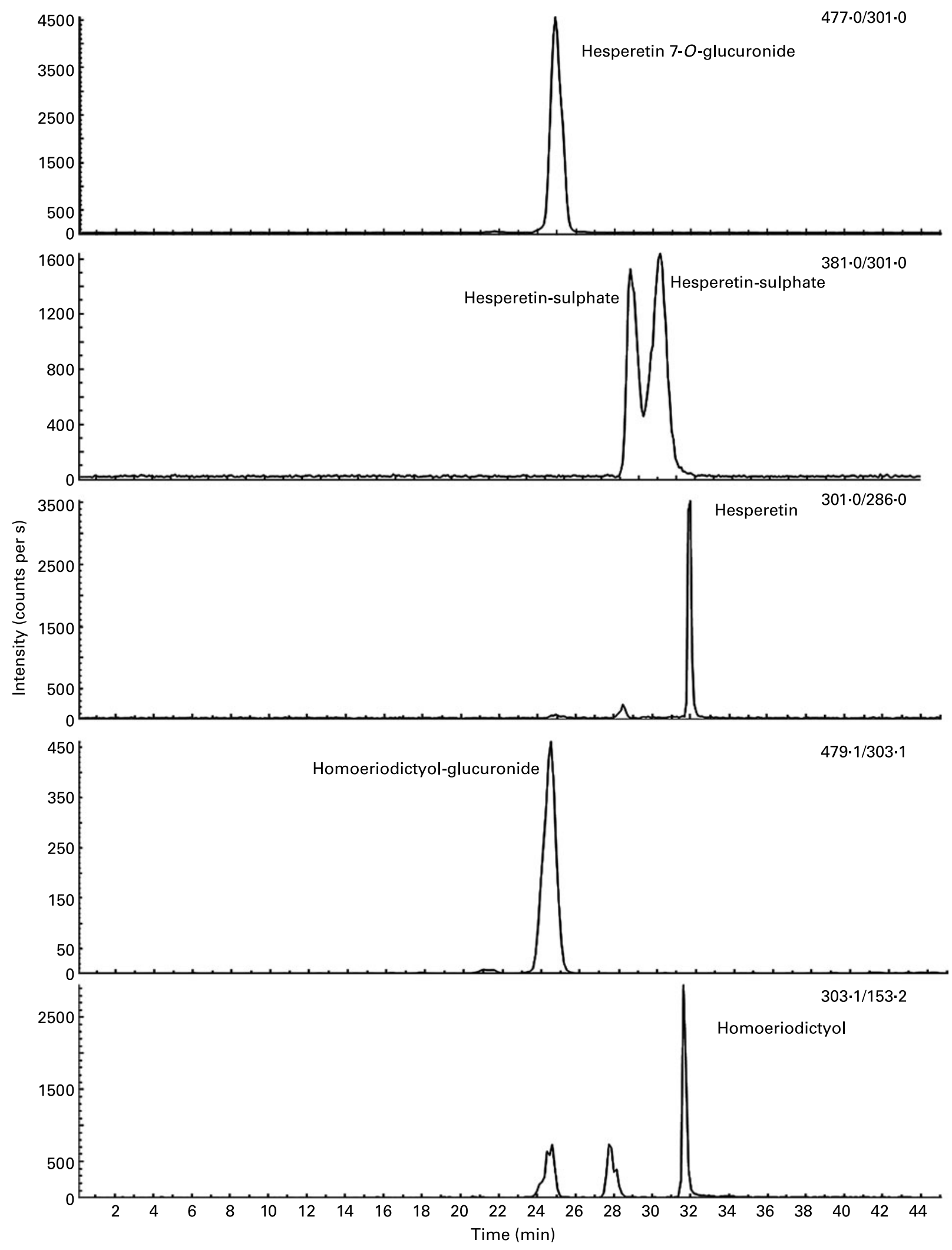

Fig. 4. Liquid chromatography-electrospray ionisation-MS/MS mass spectra for hesperidin ( $\mathrm{Hp})$ and hesperetin-7-glucoside (H-7-glc) circulating metabolites. 
Table 3. Identification and quantification of hesperidin $(\mathrm{Hp})$ and hesperetin-7-glucoside ( $\mathrm{H}-7$-glc) metabolites in plasma analysed by liquid chromatography-electrospray ionisation-MS/MS

\begin{tabular}{lccccc}
\hline & \multicolumn{5}{c}{ Respective proportion of metabolites (percentage of total flavanones) } \\
\cline { 2 - 6 } & Hesperetin & Hesperetin-7-O-glucuronide & Hesperetin sulphate & Homoeriodictyol & Homoeriodictyol glucuronides \\
\hline $0.25 \mathrm{Hp}$ & 1.9 & 54.0 & 2.7 & 3.0 & 38.4 \\
$0.5 \mathrm{Hp}$ & 3.8 & 55.0 & 2.0 & 2.7 & 36.5 \\
$0.25 \mathrm{H}-7-$ glc & 1.5 & 60.0 & 38.5 & - & - \\
$0.5 \mathrm{H}-7-$ glc & 4.7 & 66.0 & 29.3 & - & - \\
\hline
\end{tabular}

pretreated with $\alpha$-rhamnosidase, plasma hesperetin sulphates (38.5 and $29.3 \%$ for both 0.25 and $0.5 \%$ doses, respectively; Table 3) were much higher than in the groups fed Hp in untreated orange juice. Hesperetin-3'-O -glucuronide, homoeriodictyol-4' $O$-glucuronide and homoeriodictyol-7-O-glucuronide were all absent from plasma in the group fed $\mathrm{H}-7$-glc.

\section{Discussion}

Presently, the OVX rat model is the most widely used animal model for studying postmenopausal-induced osteopenia ${ }^{(27)}$. We, and others, have previously reported that OVX-induced osteopenia was more severe in adult (6-9 months old) than growing (3-6-months old) rats, leading to greater loss of trabecular and cortical bone density as well as femoral bone strength $^{(1,28)}$. In the present study, we chose the adult rat as the present model for postmenopausal osteoporosis. As expected, ovariectomy, validated by uterine atrophy (Table 1), induced significant osteopenia at all femoral sites (T-BMD: $-7.4 \%$ OVX control v. SH; Fig. 1). Both cancellous and cortical bones were affected by bone loss. Femoral mechanical properties, determined by the femoral diaphysis failure load, were impaired by OVX (Table 2). Similar to that observed in postmenopausal women ${ }^{(29)}$, bone turnover was increased following OVX as indicated by a higher urinary DPD excretion and an increase in plasma levels of OC in OVX rats compared with SH (Fig. 2). Thus, the OVX adult rat used in the present study may be considered as an appropriate model for postmenopausal bone loss ${ }^{(30,31)}$.

In accordance with our previous study in young and adult OVX rats ${ }^{(16)}$, the data shown here confirm that consumption of $\mathrm{Hp}$ at $0.5 \%$ in the diet leads to a prevention of OVX-induced bone loss in adult rats, without a uterotrophic effect and with no effect on body weight. The BMD-sparing effect (T-BMD: $+7.0 \%$ v. OVX control; Fig. 1) was associated with a partial inhibition of OVX-induced DPD excretion $(-41.7 \%$ v. OVX; Fig. 2$)$ and a significant improvement in femoral failure load $(+10.0 \% v$. OVX; Table 2$)$. These data on the bone-sparing effects of $\mathrm{Hp}$ are further supported by the work of Chiba et al. ${ }^{(15)}$, who showed that $0.5 \% \mathrm{Hp}$ in the diet of young OVX mice was able to inhibit OVX-induced trabecular bone loss and thickness at the femoral metaphysis accompanied by a reduction in the number of osteoclasts, and Deyhim et al. ${ }^{(17)}$, who showed that citrus juice prevented bone loss in male orchidectomised senescent rats accompanied by a decrease in urinary excretion of hydroxyproline.

The new finding reported here is the greater efficiency of $\mathrm{H}-7$-glc compared with native $\mathrm{Hp}$, in preventing OVX-induced bone loss. Indeed, in terms of hesperetin aglycone equivalents, the doses of $0.25 \% \mathrm{H}-7$-glc and $0.5 \% \mathrm{Hp}$ showed similar protection against OVX-induced bone loss as shown by the improvement of BMD (+6.6 and $+7.0 \%$, respectively, for T-BMD compared with OVX controls, $P<0.05$; Fig. 1) and femoral failure load $(+7.4$ and $+10.0 \%$, respectively, compared with OVX controls, $P<0.05$; Table 2). The higher dose $(0.5 \%)$ of $\mathrm{H}$-7-glc did not result in any further improvement in BMD or femoral failure load, suggesting a threshold effect at a certain concentration of hesperetin. The BMD and femoral failure load changes were accompanied by a partial but significant inhibition of the OVX-induced bone resorption (Fig. 2(b)). However, there was not a doseresponse relationship of $\mathrm{Hp}$ or $\mathrm{H}-7$-glc to this marker, and no significant change was seen in the OVX-induced OC levels.

Fig. 5 summarises the main findings on the metabolism of $\mathrm{Hp}$ based on literature data and our new finding reported here. Hp intake by human subjects showed that hesperetin is absorbed in the colon after cleavage of the rhamnoglucoside moiety. Subsequently, the main circulating forms observed are conjugates of hesperetin with glucuronic acid and/or sulphate ${ }^{(19)}$. After enzymatic removal of the rhamnose sugar to give H-7-glc, the level of hesperetin in blood from healthy volunteers was increased ${ }^{(20)}$. The results reported here in rats also show that the nature of the sugars attached to hesperetin is critical in determining the site and extent of absorption, and, in agreement with the human studies, show that more is found in the plasma after consumption of H-7-glc compared with Hp (Fig. 3(a)). Indeed, the circulating plasma levels were approximately 2-fold higher in the $\mathrm{H}$-7-glc groups compared with the Hp groups at each dose, and the $0.25 \%$ H-7-glc dose resulted in the same plasma hesperetin levels as the $0.5 \% \mathrm{Hp}$ dose (Fig. 3(a)). These higher plasma levels were accompanied by significantly higher urinary excretion levels of hesperetin in the H-7-glc groups (Fig. 3(b)). Comparing equivalent doses of $\mathrm{Hp}$ and $\mathrm{H}-7$-glc, it was clear that the $\alpha$-rhamnosidase treatment of orange juice converting $\mathrm{Hp}$ to $\mathrm{H}$-7-glc resulted in enhanced relative urinary excretion of hesperetin (Fig. 3(b)). All together, these results show an increased bioavailability of H-7-glc compared with Hp.

In addition to the higher overall levels of hesperetin in plasma after consumption of $\mathrm{H}-7$-glc, the profile of conjugates and metabolites is changed (Table 3). Shifting the absorption from the colon to the small intestine increases the percentage of sulphated metabolites. This could be due to the site of absorption and/or to flavanone-mediated induction of UDPglucuronosyltransferases $\left(\right.$ UGT1A1) ${ }^{(32)}$. The change in absorption site from the colon to the small intestine also decreased the formation of eriodictyol conjugates to undetectable levels. The latter are derived from microbial metabolism, consistent with this altered site of absorption, since rat gut microflora is able to transmethylate hesperetin 


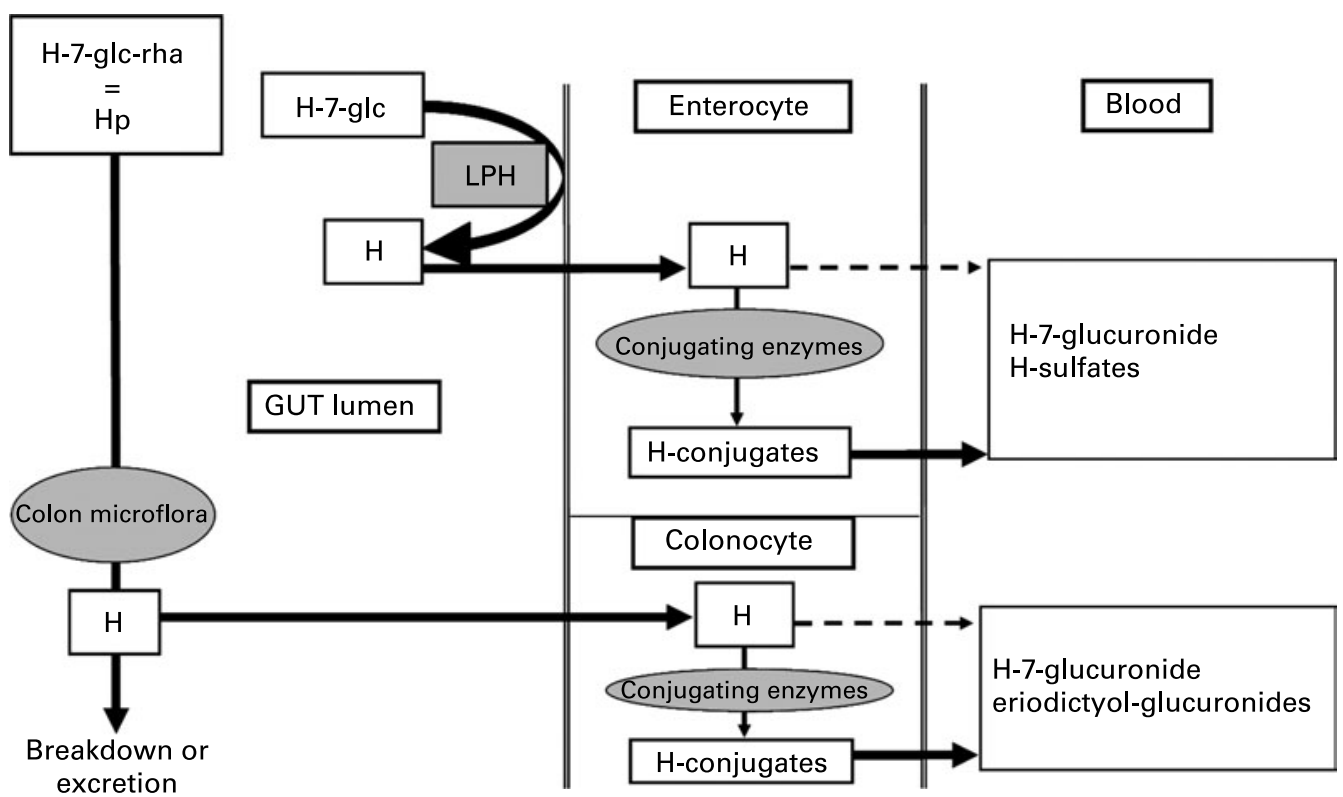

Fig. 5. Differences in metabolism of hesperidin ( $\mathrm{Hp}$; hesperetin-7-glucose-rhamnose, H-7-glc-rha) compared with hesperetin-7-glucoside (H-7-glc): conjugation profiles and sites of absorption. $\mathrm{H}$, hesperetin; LPH, lactate phlorizin hydrolase.

to form homoeriodictyol (from $-\mathrm{OCH}_{3}$ at $4^{\prime}$-position and $-\mathrm{OH}$ at $3^{\prime}$-position in hesperetin to $-\mathrm{OCH}_{3}$ at $3^{\prime}$-position and $-\mathrm{OH}$ at $4^{\prime}$-position in homoeriodictyol $)^{(33)}$. Rat colonic microflora can also hydroxylate and/or dehydroxylate and methylate naringenin to hesperetin and isosakuranetin ${ }^{(34)}$.

\section{Conclusions and perspectives}

In conclusion, in the present study, both $\mathrm{Hp}$ and $\mathrm{H}$-7-glc were able to elicit protective effects on bone loss in adult animals, probably via an inhibition of bone resorption and without uterotrophic effects. The $\mathrm{H}$-7-glc form achieved the same level of protection as $\mathrm{Hp}$ but at the lower dose of $0.25 \%$ compared with $0.5 \% \mathrm{Hp}$, but showed no further increase in bone protection at $0.5 \%$ in the diet, suggesting a threshold effect linked to hesperetin availability. Taken together with the analysis of the hesperetin plasma and urine metabolites, these results may be explained by the difference in bioavailability between these two compounds. The enzymatic conversion of Hp to H-7-glc leading to both a higher level of total hesperetin at the lower dose and an increased level of hesperetin sulphates could contribute to the more efficient bone-sparing effect of $\mathrm{H}-7$-glc. On the other hand, the absence of the homoeriodictyol metabolites following $\mathrm{H}$-7-glc consumption suggests that these metabolites are not involved in the bone-sparing effect of $\mathrm{Hp} . \mathrm{Hp}$ or H-7-glc metabolites could reach bone tissues, or possibly be deconjugated at the target site, as reported for other conjugated flavonoids in target tissues under stress conditions ${ }^{(35,36)}$.

The exact molecular mechanism of action of hesperetin or its metabolites on bone cells is not yet elucidated, although some insight from its antioxidant and anti-inflammatory properties suggest that at least some of the important pathways include $\mathrm{NF}-\kappa \mathrm{B}$ and its related signal transduction pathways, $\mathrm{NF}-\kappa \mathrm{B}$-inducing kinase/inhibitor of NF-кB kinase, extracellular signal-regulated kinase, p38 and c-Jun N-terminal kinase as well as the redox-regulating transcription factors thioredoxin/ redox factor- $1^{(37)}$. As these pathways are also implicated in the bone resorption process, they may help to explain the antiresorptive effect of Hp. The higher bioavailability of hesperetin from H-7-glc, together with the different metabolite profile, may strongly influence the cellular signalling responses in bone and other tissues and may help to explain the higher efficiency in bone protection by H-7-glc shown here.

The identification of a commonly consumed polyphenol, $\mathrm{Hp}$, together with its more potent metabolite, H-7-glc, which help to prevent OVX bone loss, opens up perspectives for the dietary management of women's health in relation to menopause or osteopenia.

\section{Acknowledgements}

The authors gratefully acknowledge the scientific collaboration and financial support of Nestec SA (Nestlé Research Center, Department of Nutrition and Health, Lausanne, Switzerland). The authors declare that there is no conflict of interest. M. N. H. designed and supervised aspects of the whole study. P. L., M. J. D., A. T. and V. C. contribute to animal study and bone measurements. C. M. and A. G.-I. performed HPLC analyses. D. B. kindly provided synthesised hesperetin-7- $O$-glucuronide. V. H. conducted the data collection, the statistical analysis and wrote the manuscript. E. O., G. W., C. M., W. C. and I. L. N. critically reviewed the manuscript.

\section{References}

1. Horcajada-Molteni MN, Crespy V, Coxam V, et al. (2000) Rutin inhibits ovariectomy-induced osteopenia in rats. $J$ Bone Miner Res 15, 2251-2258.

2. Muhlbauer RC, Lozano A \& Reinli A (2002) Onion and a mixture of vegetables, salads, and herbs affect bone resorption in the rat by a mechanism independent of their base excess. J Bone Miner Res 17, 1230-1236. 
3. Liu ZP, Li WX, Yu B, et al. (2005) Effects of trans-resveratrol from Polygonum cuspidatum on bone loss using the ovariectomized rat model. J Med Food 8, 14-19.

4. Setchell KDR \& Lydeking-Olsen E (2003) Dietary phytoestrogens and their effect on bone: evidence from in vitro and in vivo, human observational, and dietary intervention studies. Am J Clin Nutr 78, 593S-599S.

5. Potter SM, Baum JA, Teng HY, et al. (1998) Soy protein and isoflavones: their effects on blood lipids and bone density in postmenopausal women. Am J Clin Nutr 68, 1375S-1379S.

6. Chen YM, Ho SC, Lam SSH, et al. (2003) Soy isoflavones have a favorable effect on bone loss in Chinese postmenopausal women with lower bone mass: a double-blind, randomized, controlled trial. J Clin Endocrinol Metabol 88, 4740-4747.

7. Messina M, Ho S \& Alekel DL (2004) Skeletal benefits of soy isoflavones: a review of the clinical trial and epidemiologic data. Curr Opin Clin Nutr Metab Care 7, 649-658.

8. Cassidy A, Albertazzi P, Nielsen IL, et al. (2006) Critical review of health effects of soyabean phyto-oestrogens in postmenopausal women. Proc Nutr Soc 65, 76-92.

9. Scalbert A, Morand C, Manach C, et al. (2002) Absorption and metabolism of polyphenols in the gut and impact on health. Biomed Pharmacother 56, 276-282.

10. Gil-Izquierdo A, Gil MI, Ferreres F, et al. (2001) In vitro availability of flavonoids and other phenolics in orange juice. $J$ Agric Food Chem 49, 1035-1041.

11. Knekt P, Kumpulainen J, Jarvinen R, et al. (2002) Flavonoid intake and risk of chronic diseases. Am J Clin Nutr 76, $560-568$

12. Galati EM, Monforte MT, Kirjavainen S, et al. (1994) Biological effects of hesperidin, a citrus flavonoid. (Note I): antiinflammatory and analgesic activity. Farmaco 40, 709-712.

13. Miyake Y, Yamamoto K, Tsujihara N, et al. (1998) Protective effects of lemon flavonoids on oxidative stress in diabetic rats. Lipids 33, 689-695.

14. Garg A, Garg S, Zaneveld LJ, et al. (2001) Chemistry and pharmacology of the citrus bioflavonoid hesperidin. Phytother Res 15, 655-669.

15. Chiba H, Uehara M, Wu J, et al. (2003) Hesperidin, a citrus flavonoid, inhibits bone loss and decreases serum and hepatic lipids in ovariectomized mice. J Nutr 133, 1892-1897.

16. Horcajada MN, Habauzit V, Trzeciakiewicz A, et al. (2008) Hesperidin inhibits ovariectomized-induced osteopenia and shows differential effects on bone mass and strength in young and adult intact rats. J Appl Physiol 104, 648-654.

17. Deyhim F, Garica K, Lopez E, et al. (2006) Citrus juice modulates bone strength in male senescent rat model of osteoporosis Nutrition 22, 559-563.

18. Gil-Izquierdo A, Riquelme MT, Porras I, et al. (2004) Effect of the rootstock and interstock grafted in lemon tree (Citrus limon (L.) Burm.) on the flavonoid content of lemon juice. J Agric Food Chem 52, 324-331.

19. Manach C, Morand C, Gil-Izquierdo A, et al. (2003) Bioavailability in humans of the flavanones hesperidin and narirutin after the ingestion of two doses of orange juice. Eur J Clin Nutr 57, 235-242.

20. Nielsen IL, Chee WS, Poulsen L, et al. (2006) Bioavailability is improved by enzymatic modification of the citrus flavonoid hesperidin in humans: a randomized, double-blind, crossover trial. J Nutr 136, 404-408.

21. Graefe EU, Wittig J, Mueller S, et al. (2001) Pharmacokinetics and bioavailability of quercetin glycosides in humans. $J$ Clin Pharmacol 41, 492-499.

22. Pastoureau P, Chomel A \& Bonnet J (1995) Specific evaluation of localized bone mass and bone loss in the rat using dualenergy X-ray absorptiometry subregional analysis. Osteoporos Int 5, 143-149.

23. Turner CH \& Burr DB (1993) Basic biomechanical measurements of bone: a tutorial. Bone 14, 595-608.

24. Robins SP (1994) Biochemical markers for assessing skeletal growth. Eur J Clin Nutr 48, Suppl. 1, S199-S209.

25. Cook JG (1975) Factors influencing the assay of creatinine. Ann Clin Biochem 12, 219-232.

26. Matsumoto H, Ikoma Y, Sugiura M, et al. (2004) Identification and quantification of the conjugated metabolites derived from orally administered hesperidin in rat plasma. J Agric Food Chem 52, 6653-6659.

27. Kalu DN (1991) The ovariectomized rat model of postmenopausal bone loss. Bone Miner 15, 175-191.

28. Picherit C, Chanteranne B, Bennetau-Pelissero C, et al. (2001) Dose-dependent bone-sparing effects of dietary isoflavones in the ovariectomised rat. Br J Nutr 85, 307-316.

29. Riggs BL, Khosla S, Atkinson EJ, et al. (2003) Evidence that type I osteoporosis results from enhanced responsiveness of bone to estrogen deficiency. Osteoporos Int 18, 728-733.

30. Omi N \& Ezawa I (1995) The effect of ovariectomy on bone metabolism in rats. Bone 17, $163 \mathrm{~S}-168 \mathrm{~S}$.

31. Cai DJ, Zhao YD, Glasier J, et al. (2005) Comparative effect of soy protein, soy isoflavones, and 17 beta-estradiol on bone metabolism in adult ovariectomized rats. $J$ Bone Miner Res 20, $828-839$.

32. Walle UK \& Walle $\mathrm{T}$ (2002) Induction of human UDP-glucuronosyltransferase UGT1A1 by flavonoids-structural requirements. Drug Metab Dispos 30, 564-569.

33. De Eds F, Booth AN \& Jones TF (1957) Methylation and dehydroxylation of phenolic compounds by rats and rabbit. $J$ Biol Chem 225, 615-621.

34. Silberberg M, Gil-Izquierdo A, Combaret L, et al. (2006) Flavanone metabolism in healthy and tumor-bearing rats. Biomed Pharmacother 60, 529-535.

35. Yuan L, Wagatsuma C, Sun B, et al. (2003) The role of betaglucuronidase in induction of apoptosis by genistein combined polysaccharide (GCP) in xenogeneic mice bearing human mammary cancer cells. Ann N Y Acad Sci 1010, 347-349.

36. Shimoi $\mathrm{K} \&$ Nakayama $\mathrm{T}$ (2005) Glucuronidase deconjugation in inflammation. Methods Enzymol 400, 263-272.

37. Kim JY, Jung KJ, Choi JS, et al. (2006) Modulation of the age-related nuclear factor-kappaB (NF-kappaB) pathway by hesperetin. Aging Cell 5, 401-411. 